L'établissement rural de La Tène finale du «Vau Blanchard » à Lavernat (Sarthe) : vers un plan type de la ferme gauloise?

The Late La Tene "Vau Blanchard" farmstead of Lavernat (Sarthe)towards a Gaulish farm type-plan?

Gérard Guillier, Jean Brodeur et Emmanuelle Coffineau

\title{
(2penEdition Journals
}

Édition électronique

URL : https://journals.openedition.org/rao/841

DOI : 10.4000/rao.841

ISBN : 978-2-7535-1609-0

ISSN : 1775-3732

Éditeur

Presses universitaires de Rennes

Édition imprimée

Date de publication : 31 décembre 2009

Pagination : 117-134

ISBN : 978-2-7535-1086-9

ISSN : 0767-709X

Référence électronique

Gérard Guillier, Jean Brodeur et Emmanuelle Coffineau, «L'établissement rural de La Tène finale du "Vau Blanchard » à Lavernat (Sarthe) : vers un plan type de la ferme gauloise? », Revue archéologique de l'Ouest [En ligne], 26 | 2009, mis en ligne le 31 décembre 2011, consulté le 22 août 2022. URL http://journals.openedition.org/rao/841 ; DOI : https://doi.org/10.4000/rao.841 


\title{
L'établissement rural de La Tène finale du «Vau Blanchard » à Lavernat (Sarthe) : vers un plan type de la ferme gauloise?
}

\author{
The Late La Tene "Vau Blanchard" farmstead of Lavernat (Sarthe) \\ towards a Gaulish farm type-plan?
}

\author{
Gérard Guillier*, Jean Brodeur ${ }^{* *}$ et Emmanuelle Coffineau **
}

\begin{abstract}
Résumé : Sur la commune de Lavernat, dans le sud du département de la Sarthe, la campagne de prospection archéologique systématique effectuée sur le tracé de la future autoroute A 28, a permis au niveau de l'échangeur de Montabon, près de Château-du-Loir, de mettre en évidence un enclos de plan trapézoïdal d'une base de $101 \mathrm{~m}$ pour une hauteur de $77 \mathrm{~m}$, circonscrivant une surface de $6500 \mathrm{~m}^{2}$. À ce jour, il s'agit d'une des rares fermes indigène de La Tène finale fouillée exhaustivement dans le département de la Sarthe.

Cette ferme est matérialisée au sol par des structures fossoyées arasées. Le décapage mécanique intégral de l'aire interne y a révélé un seul bâtiment d'habitat complété d'un grenier et d'un puits à eau. Quelques centaines de tessons, des plaques de foyers et des meules constituent l'essentiel des vestiges matériels exhumés tandis que de rares vestiges du $\mathrm{I}^{\text {er }}$ siècle de notre ère matérialisent l'abandon du site. De nombreux établissements ruraux très comparables permettent d'évoquer un plan-type au sein des découvertes effectuées dans l'ouest de la Gaule.
\end{abstract}

\begin{abstract}
Near Chatteau-du-Loir in the south of Sarthe, in the Commune of Lavernat, a systematic archaeological survey carried out along the planned route of the $A$ 28, uncovered, at the Montabon junction, has uncovered a trapezoid enclosure $101 \mathrm{~m}$ by $77 \mathrm{~m}$, encompassing an area of 6500 sq. $m$. This thoroughly excavated site is a rare example in the Sarthe of a "native" farmstead dating from the Late La Tene period.

The settlement appears as eroded earthworks. Complete mechanical scraping of the interior revealed a single domestic building, a storage area and a well. Several hundred shards, some hearths slabs and querns are the main associated finds. A very few remains dating from the Ist century AD indicate when the site was finally abandoned. Many quite similar farmsteads suggest that a typical layout may emerge from all the discoveries yet made in Western Gaul.
\end{abstract}

Mots clés : La Tène finale, enclos trapézoïdal, établissement rural, bâtiments sur poteaux, céramiques, terres cuites, meules.

Key words: Late La Tene, trapezoidal enclosure, farmstead, post building, pottery, fired clay, querns.

\section{INTRODUCTION}

Le site du Vau Blanchard sur la commune de Lavernat dans le sud du département de la Sarthe ${ }^{1}$ a été découvert lors des pros- pections archéologiques effectuées dans le cadre du futur tracé de l'autoroute A 28, au niveau de l'échangeur de Montabon, un peu à l'ouest de la ville de Château-du-Loir (fig. 1). Mis en

* INRAP Grand-Ouest et UMR 8546 CNRS/ENS Paris.

** INRAP Grand-Ouest-37 rue du Bignon, 35577 Cesson-Sévigné Cedex.

1. $\mathrm{N}^{\circ}$ d'entité archéologique : 72.160.0004; références cadastrales (année 2000) : section D3, parcelle $\mathrm{n}^{\circ} 482$; coordonnées Lambert : $\mathrm{X}=451901, \mathrm{Y}$ = 300807; altitude NGF : 98,5 m; surface totale décapée : $12000 \mathrm{~m}^{2}$ environ. NB : Le nom attribué au site( Le Vau Blanchard») n’est pas un lieu-dit situé de la commune de Lavernat mais celui d'une ferme sur la commune voisine de Montabon et correspond au toponyme le plus proche (fig. 3). 

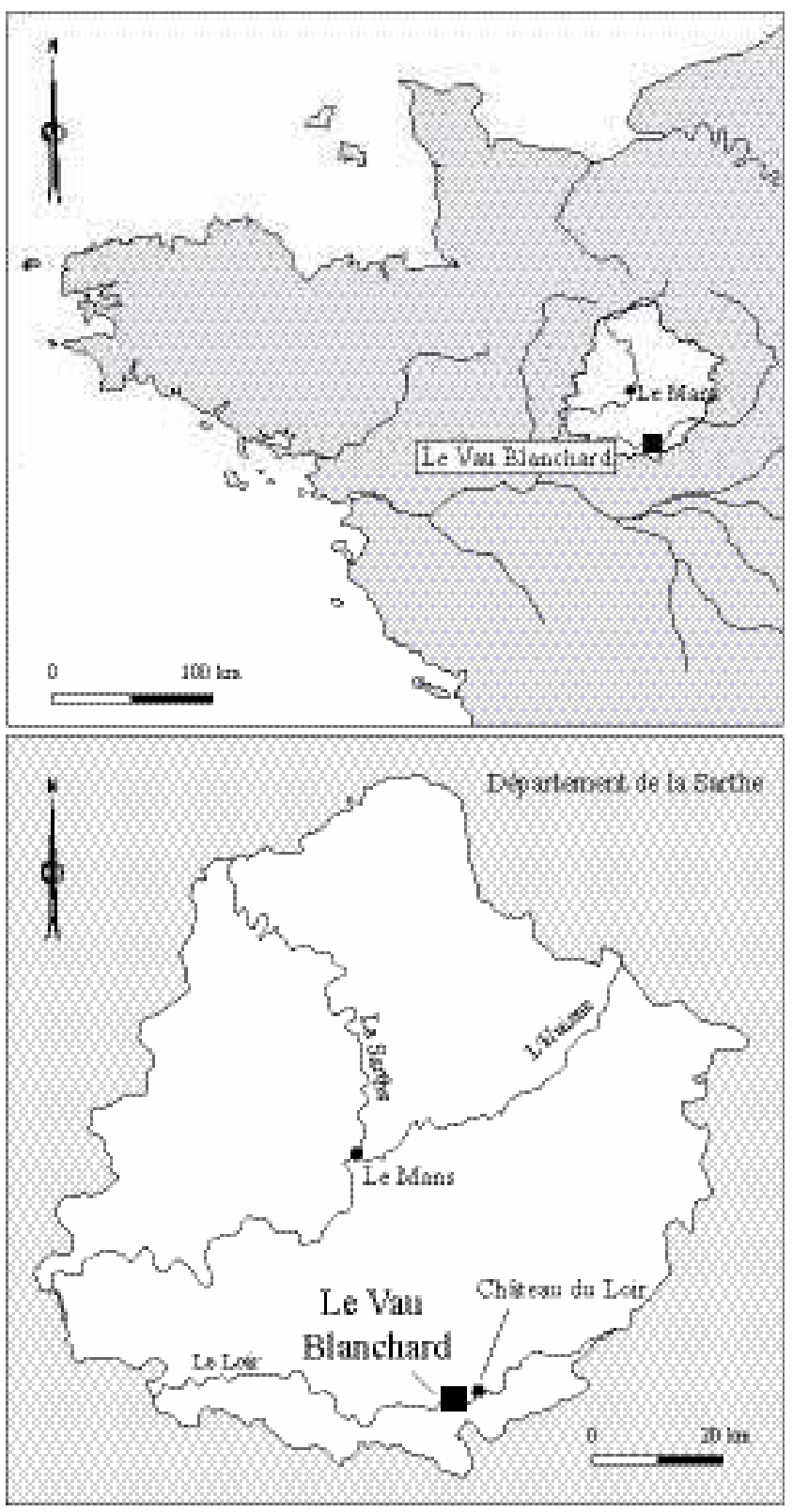

Figure 1 : Lavernat, Le Vau Blanchard : Localisation du site au sud du département de la Sarthe.

Figure 1: Location of the site in the south of dept. Sarthe.

évidence lors de sondages de diagnostic archéologique (Gallien, 2004), il a été fouillé par J. Brodeur (2006). Ce site se trouve en limite sud de la cité des Aulerques Cénomans (fig. 2), au plus près du territoire andécave. Le mobilier recueilli a permis d'identifier deux périodes chronologiques : la fin du deuxième âge du Fer et le début de l'époque gallo-romaine. La fouille avait pour but de reconnaître l'intégralité de la structure trapézoïdale, d'essayer de la caractériser et si possible d'en préciser la chronologie (Brodeur, 2006). Selon les prescriptions du Service régio- nal de l'Archéologie des Pays de la Loire, un décapage extensif fut opéré, suivi d'une fouille manuelle classique, avec les adaptations inhérentes à la nature des vestiges découverts. Les opérations de terrain se sont déroulées du 20 juin au 31 août 2004. Après un bref aperçu du contexte historique et archéologique, nous allons déterminer l'organisation du site, en présenter le mobilier et finir par une mise en perspective des résultats.

\section{Présentation générale}

\section{Topographie et environnement}

Le gisement de Lavernat occupe un plateau légèrement ondulé à une centaine de mètres d'altitude. Ce plateau est tranché à $1,6 \mathrm{~km}$ au sud de l'enclos par la rivière du Loir. Nous passons en moins de $400 \mathrm{~m}$ d'une altitude de 101 à $44 \mathrm{~m}$, ponctuellement la pente atteint $25 \%$ (fig. 3). Le site archéologique domine la tête d'un petit vallon sec orienté est-ouest, qui se trouve à cheval sur les communes de Lavernat et de Montabon. Le gisement se développe sur une légère pente ouest-est, un peu en contrebas d'un petit promontoire. Son altitude varie d'environ $99,7 \mathrm{~m}^{2}$ au sudouest à 96,3 m au nord-est (fig. 4); d'est en ouest, les pentes observées y varient de 1,7 à $2,9 \%$ et, du sud vers le nord, de 0,7 à 2,2 \%. La profondeur d'enfouissement des vestiges est peu importante, environ 0,40 à $0,50 \mathrm{~m}$. Le fond du vallon sec se trouve au sud du site à une altitude de $80 \mathrm{~m}$ et le haut du plateau, au nord, à une altitude de $114 \mathrm{~m}$.

Les sédiments tertiaires qui forment le substrat du site appartiennent à l'Éocène continental (e de la carte géologique) et sont constitués d'une argile de couleur jaune à rouille. Plus haut sur la pente, on remarque un fin placage de Limons de plateau (LP), tandis que plus bas, on observe des argiles issues de l'altération des calcaires crétacés $(\mathrm{Rc})$. Le fond du vallon est marqué de matériaux soliflués issus de Rc et de colluvions de fond de vallon (Manivit et al., 1998).

\section{Un contexte archéologique pauvre}

La carte archéologique de Lavernat était, jusqu'à cette découverte, totalement dépourvue d'indices d'occupations anciennes et la Carte archéologique de la Gaule offre une même indigence (Bouvet, 2001, $\mathrm{n}^{\circ} 160$ ). Les plus proches témoignages se situant à Aubigné Racan, à quelques kilomètres à l'ouest : une installation indigène de nature indéterminée a pu y être mise en évidence, à laquelle se substitue une agglomération antique pourvue d'une parure monumentale :

2. Ces cotes, ainsi que celles portées sur les fig. 4 à 7 , ont été prises après le décapage mécanique du site, qui a porté sur une épaisseur d'environ $0,4-0,5 \mathrm{~m}$. 


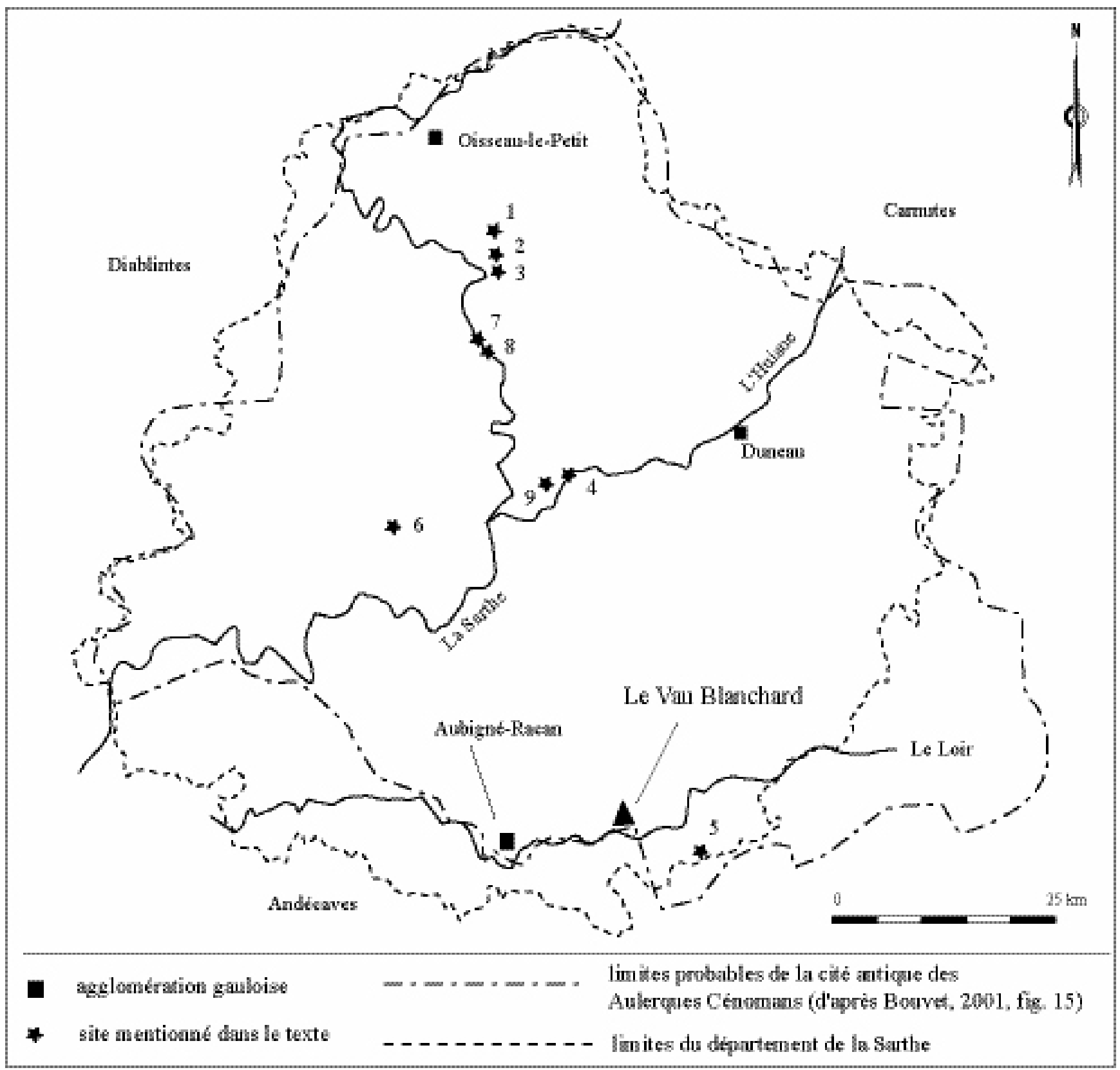

Figure 2 : Limites probables de la cité antique des Aulerques Cénomans et localisation des quelques sites sarthois mentionnés dans le texte. 1, 2 et 3 : La Fosse, La Gaudine et La Pièce de Bildoux à Vivoin; 4 : Le Grand Aunay à Yvré-l'Évêque; 5 : Beauregard à Dissé-sous-Courcillon; 6 : Le Tertre à Souligné-Flacé; 7 : Les Petites Landes à Teillé; 8 : Les Rues Vertes à Saint-Jean-D’Assé; 9 : Le Ruisseau à Yvré-L'Évêque.

Figure 2: Probable limits of the Antique Aulerci Cenomani territory and location of some mentionned sites.

théâtre, thermes, temple et forum (ibid.). Sur la même commune, des vestiges de La Tène finale forment un complexe fortifié occupé dès le premier âge du Fer sur la butte de Vau (ibid., p. 143), tandis que, du site de la Grande Pâture, proviennent des pièces d'armement datables $\mathrm{du}_{\mathrm{IV}}^{\mathrm{e}}$ au $\mathrm{II}^{\mathrm{e}}$ siècles av. J.-C. (ibid., p. 146-147). Quelques monnaies gauloises jalonnent le tracé du Loir : Aubigné-Racan, Vaas, Châteaudu-Loir, la Chartre-sur-le-Loir et Poncé-sur-le-Loir (ibid., fig. 12) et matérialisent un axe de " circulation " qui passe non loin au sud du Vau Blanchard. Les indices qui ont permis de dresser une carte des limites de la cité cénomane supposent qu'à l'ouest de Château-du-Loir, la frontière avec les Andécaves (région d'Angers) devait s'y confondre avec le cours du Loir (ibid., p. 80-81) (fig. 2). Le site de Lavernat est au plus près de la limite sud de la cité des Aulerques Cénomans, au contact de leurs voisins. Enfin, une voie de 


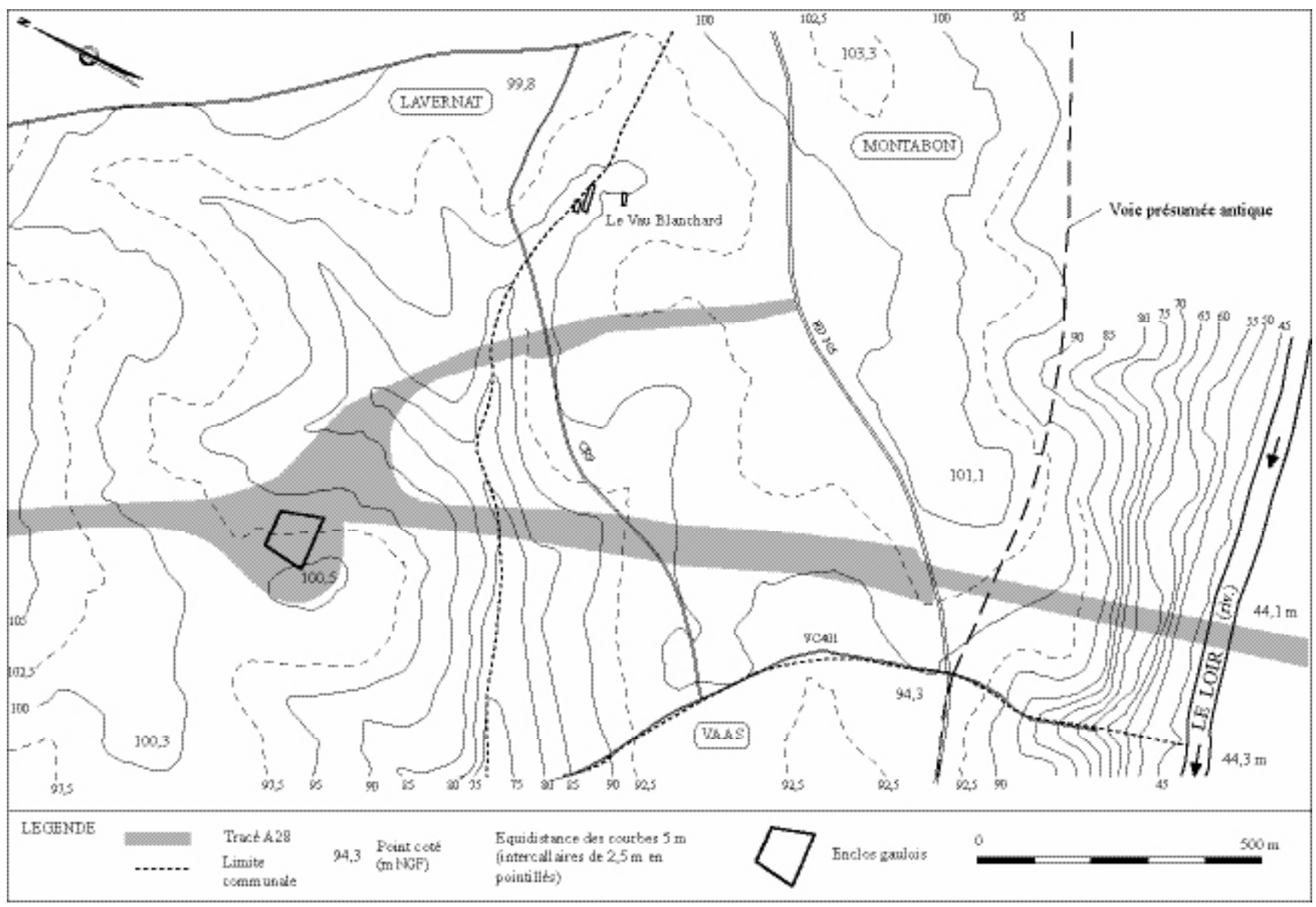

Figure 3 : Le Vau Blanchard : l'environnement topographique du site, à $700 \mathrm{~m}$ au sud-ouest de la ferme actuelle. Figure 3: Topographical surrounding of the site, some $700 \mathrm{~m}$ south-west of today's farm.

crête vraisemblablement antique passerait à environ $1100 \mathrm{~m}$ au sud du site (fig. 3) (Bouton, 1947, p. 186-187).

En dernier lieu notons que ce secteur de la commune de Lavernat était jadis en partie voué à la culture de la vigne, comme l'atteste la régularité des parcelles en lanières visibles sur le cadastre du XIx siècle $^{3}$ (fig. 4) (Brodeur, 2006, fig. 1 bis).

\section{L'enclos gaulois du Vau Blanchard}

\section{Le plan de l'enclos et son fossé}

Le fossé St. 1 décrit un trapèze quasi isocèle, d'une base de $101 \mathrm{~m}$ pour une hauteur de $77 \mathrm{~m}$ (fig. 4). Ses côtés est, nord, ouest et sud mesurent respectivement, 78, 69, 78 et $101 \mathrm{~m}$,

3. Commune de Lavernat, cadastre de 1850-1851, section D des Landes, feuille 4 (Archives Départementales, cote PC\1621069). Ce plan ne présente que peu de différences par rapport au cadastre "napoléonien " 1813, (section G des Landes, première feuille) (AD, cote PCl162l012). l'ensemble circonscrivant une surface d'environ $6500 \mathrm{~m}^{2}$. Plusieurs remarques d'ordre géométrique peuvent être effectuées à partir de la simple observation du plan. En premier lieu, les médianes de ce trapèze, orientées nord-sud et estouest, trahissent une nette volonté d'orientation cardinale. En second lieu, le trapèze qui matérialise l'enclos forme la base géométrique d'un vaste triangle isocèle haut théoriquement de $232 \mathrm{~m}$. Or la hauteur du trapèze est de $77 \mathrm{~m}$, soit exactement le tiers de la hauteur de ce triangle théorique. Nous pouvons donc envisager une construction géométrique basée sur une figure simple - le triangle isocèle - et des calculs élémentaires - division par 3 - permettant par ailleurs d'obtenir une figure géométrique parfaitement équilibrée. D’autres remarques transparaissent au travers de ces données, concernant d'éventuelles unités de mesure : l'utilisation d'un pes local de $0,322 \mathrm{~m}$ peut être envisagée à partir de la hauteur du trapèze isocèle ( $77 \mathrm{~m}=2$ actus de 120 pes chacun), cette unité de base étant proche du pes de $0,326 \mathrm{~m}$ déduit de la valeur moyenne de la leuga gauloise de $2450 \mathrm{~m}$ (Dassier, 2000). 


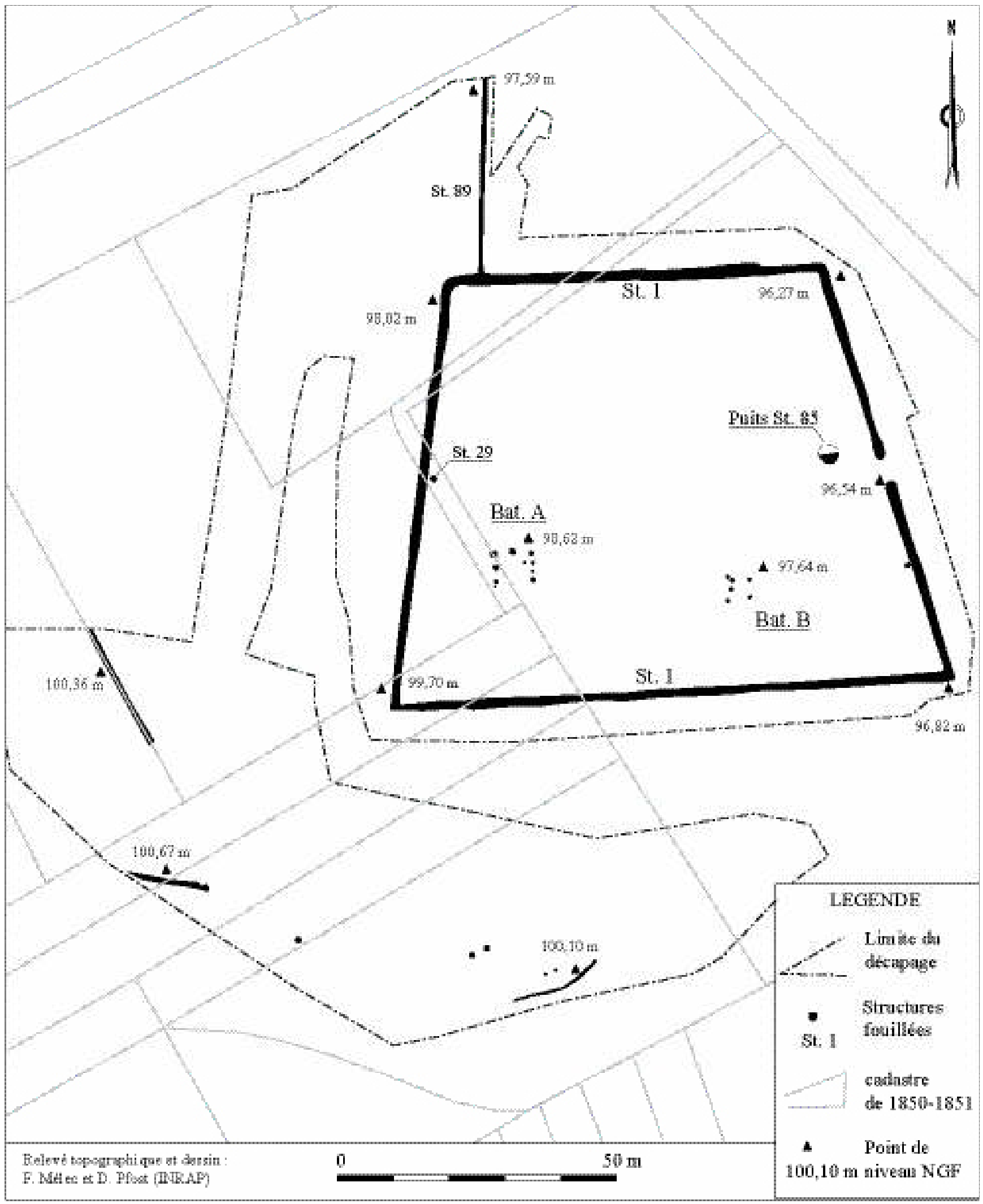

Figure 4 : Le Vau Blanchard : plan détaillé des vestiges sur le fond cadastral ancien (1850-1851). Figure 4: Detailed plan of the remains reported on the ancient cadastral map (1850-1851). 
Ces premiers éléments impliquent à notre sens, qu’au moment du creusement de la structure, le plan à obtenir bénéficiait d'un système de repérage au sol ${ }^{4}$. Les terrassiers ainsi guidés ont alors pu creuser l'ensemble du linéaire. De manière générale, le fossé St. 1, creusé dans les argiles naturelles sur 0,60 à $1 \mathrm{~m}$ de profondeur, présente un profil en forme de $\mathrm{V}$, avec cependant, quelques différences quant à la pente des bords (fig. 5 et 6 ). Ainsi, sur les sondages 47 et 77 (fig. 5), celle-ci est-elle très marquée vers un fond très étroit, tandis qu'ailleurs, le fond aplati, légèrement plus large, est complété de bords évasés ( $\mathrm{Sd} .57$ et 46, fig. 5). On constate même à plusieurs endroits, à mi-pente, une sorte de méplat plus ou moins accentué ( $\mathrm{Sd}$. 65, fig. 6), témoignant peutêtre de récurages difficiles à identifier (Sd 22, fig. 6).

Le remplissage du fossé d'enclos a livré peu d'informations. On a constaté, au fond, la présence d'un niveau hydromorphe, de texture plus ou moins argilo-sableuse et de teinte souvent grise à gris-orangé ou ocrée, contemporain du fonctionnement à ciel ouvert de la structure (fig. 5 et 6 ) : le ruissellement des eaux sur les bords compacts a entrainé cette accumulation caractéristique d'épaisseur variable où le mobilier de La Tène a tendance à se concentrer. Ensuite se remarquent des couches de stabilisation, caractéristiques des fossés qui ont fonctionné en mode ouvert (sd. 47 et 57 , US 3, fig. 5 ; sd. 31 et 40, US 2, fig. 6). Les niveaux susjacents montrent en coupe des taches de couleur différente de la masse. Un comblement, à l'aide de « mottes " vraisemblablement arrachées à un petit talus d'escarpe a sans doute donné ce type de stratigraphie; c'est dans ces horizons que le mobilier le plus récent a été recueilli. Enfin, des anomalies dans le tracé du fossé et dans le comblement, observées notamment au niveau du passage aménagé sur le côté oriental, permettent d'envisager des recreusements ponctuels (fig. 5, Sd. 47; fig. 6, Sd. 22).

Ce passage est parfaitement placé au centre du côté est, à $37 \mathrm{~m}$ des angles nord-est et sud-est, montrant là encore une organisation préalable du chantier de terrassement et l'application de règles simples de géométrie. La fouille n'a montré aucun aménagement particulier pour ce seul accès, large de quatre mètres. L'environnement, notamment la localisation de chemins ou de voies a dû être déterminante, mais leur identification reste en suspens.

Nous suggérons aussi que la forme trapézoïdale marque une adaptation à la topographie locale pour gérer au mieux l'écoulement des eaux (Guillier et al., 2005). En effet, on remarque, près de l'angle nord-ouest de l'enclos, la présence du fossé 89 (fig. 4), dont la largeur varie de 0,60 à $0,80 \mathrm{~m}$ (fig. 6). Parfaitement orienté nord-sud, ce St. 89 se connecte

4. Un autre élément vient confirmer la construction géométrique préalable du tracé du fossé d'enclos : la médiane est-ouest passe exactement au milieu du passage oriental de St. 1. au côté nord de l'enclos; il accuse une légère pente vers le nord et les couches de son remplissage sont limoneuses mais, malheureusement, aucun mobilier n'y a été mis au jour. Cette structure que nous attribuons à La Tène finale a joué un rôle d'évacuation de l'eau pour éviter sa stagnation dans le fossé St. 1. Il a pu aussi former l'amorce d'une bassecour, phénomène lié couramment aux enclos trapézoïdaux; citons par exemple les sites de Douains dans l'Eure (Varin, 2007), d'Herblay dans le Val-d'Oise (Valais, 1994) ou de Pouille en Vendée (Nillesse, 1994). Une autre possibilité, n'excluant pas les précédentes propositions, est celle d'une amorce de parcellaire.

\section{L'aire interne de l'enclos : peu de vestiges}

Le décapage intégral de l'intérieur de l'enclos a seulement mis en évidence deux bâtiments sur poteaux plantés, localisés dans sa moitié sud (fig. 4; fig. 7). L'un, le Bâtiment A, se trouve à proximité de son angle sud-ouest; le second, le Bâtiment $\mathrm{B}$, distant du premier d'une trentaine de mètres, est plus proche de l'entrée.

\section{Le bâtiment $A$}

Ce bâtiment présente un plan rectangulaire régulier (fig. 7 , en haut). Il mesure $6,5 \times 4,8 \mathrm{~m}$, soit $20 \times 15$ pes de $0,322 \mathrm{~m}$. Le rapport longueur / largeur est de $4 / 3$, une application directe $\mathrm{du}$ " théorème de Pythagore " qui permet d'envisager un traçage au sol à l'aide d'une corde à nœuds. Trois des côtés sont ponctués de trous de poteaux, parfois de taille importante; le côté sud paraît totalement ouvert mais nous pouvons suggérer que l'érosion a fait disparaître les trous les moins profonds. La surface utile de ce bâtiment $\mathrm{A}, 30 \mathrm{~m}^{2}$, entre dans la moyenne des "bâtiments vrais " tels que définis par P. Pion (1996, p. 90) dans la vallée de l'Aisne, où ils varient de 26 à $33 \mathrm{~m}^{2}$.

Pour le département de la Sarthe, aucun plan similaire n’a été relevé; nous avons seulement remarqué que certains des modules définis offrent des dimensions voisines : 7 x $4 \mathrm{~m}$ et $8 \times 5 \mathrm{~m}$ par exemple sur les sites "La Petite Némerie " ou "La Pièce de Bildoux " ̀̀ Vivoin (Maguer et al., 2003, fig. 15). Des structures assez comparables ont aussi été mises au jour en Haute-Normandie, à Tournedos-sur-Seine, Condé-sur-Iton, etc. (Dechezleprêtre et al., 2000, p. 329 à 331 et fig. 2). Un des bâtiments les plus semblables a été découvert à Avrilly dans l'Eure (Guillier et al., 2005) (fig. 7, en bas).

\section{Le bâtiment $B$}

Le plan de ce bâtiment (fig. 7 , au centre) est dessiné par six trous de poteaux de $0,60 \mathrm{~m}$ de diamètre (St. 78 à 83), définissant une surface trapézoïdale de $3,20 \mathrm{~m}$ de côté 


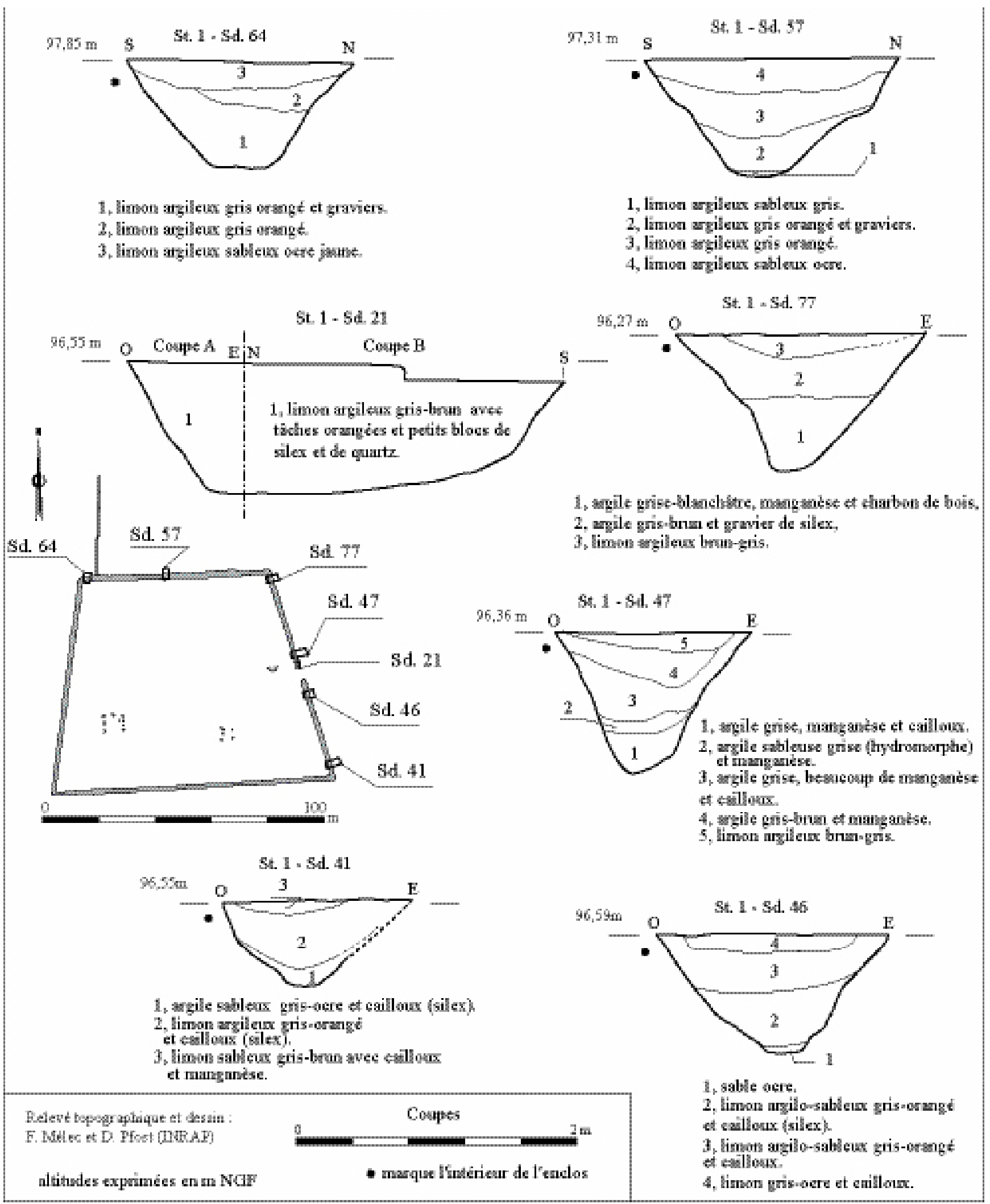

Figure 5: Le Vau Blanchard, enclos trapézoïdal : stratigraphie des côtés est et nord.

Figure 5: Trapezoidal enclosure: stratigraphy of east and north sides. 


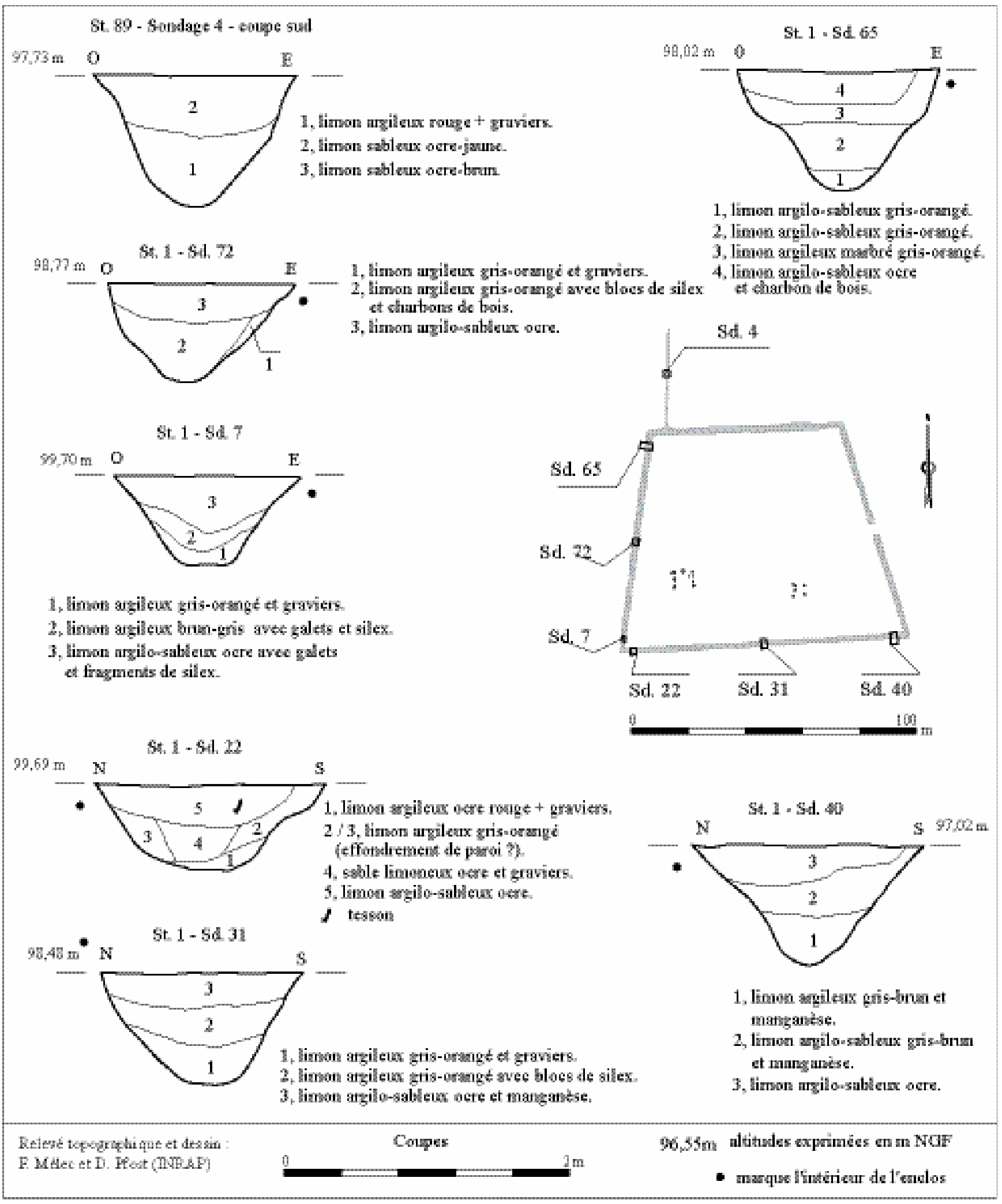

Figure 6 : Le Vau Blanchard, enclos trapézoïdal : stratigraphie des côtés ouest et sud ainsi que de l'antenne nord (St. 89). Figure 6: Trapezoidal enclosure: stratigraphy of west and south sides, plus northern antenna (St.89). 


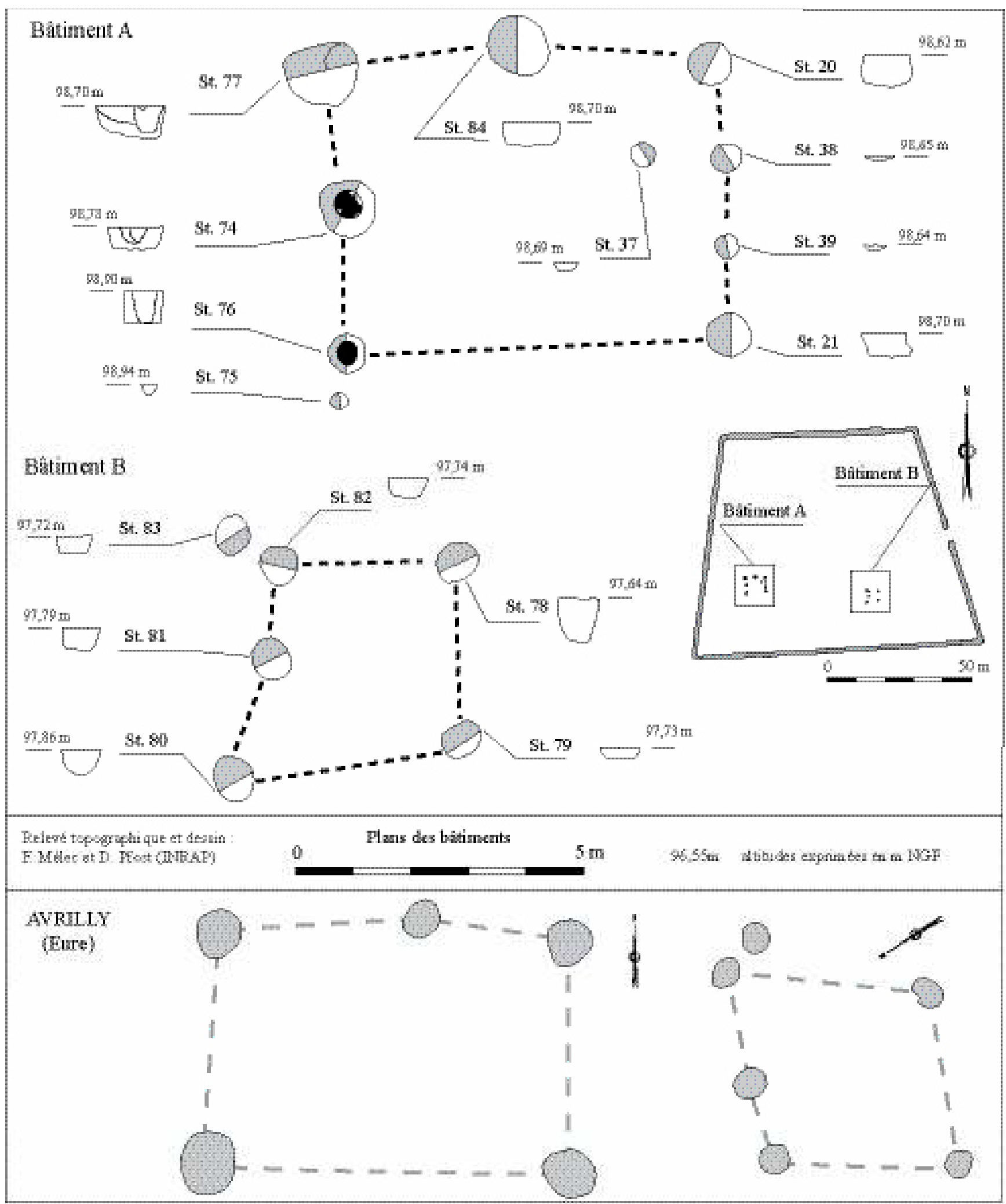

Figure 7 : Le Vau Blanchard : plans détaillés des Bâtiments A et B et, en comparaison, plans de ceux d'Avrilly dans l'Eure (même échelle).

Figure 7: Detailed plans of Buildings A and B plus, for comparison, those of of Avrilly (dept. Eure), at the same scale. 
environ. St. 80 est légèrement décalé vers le sud tandis que St. 83 paraît ajouté à l'angle nord-ouest. Les profondeurs conservées des trous occidentaux sont de 0,40 m. À l'angle nord-est, une profondeur de $0,70 \mathrm{~m}$ (St. 78), correspond à un poteau plus fort. Il y aurait ici le même phénomène que pour le Bâtiment $\mathrm{A}$ avec des renforcements à l'ouest et au nord, face à la pente.

Ce bâtiment $\mathrm{B}$ pourrait correspondre à un grenier surélevé; cependant ses dimensions le placent à la charnière, située théoriquement à $3 \mathrm{~m}$ de côté, entre les greniers surélevés et les bâtiments sur sol (Dechezleprêtre et al., 2000, p. 328). Des exemples, souvent de dimensions moindres, ont été mis au jour dans les Pays de la Loire : Vendée, LoireAtlantique, Maine-et-Loire (Nillesse, 2003), Sarthe (Maguer et al., 2003). Une forme très proche a été découverte à Avrilly dans l'Eure (Guillier et al., 2005) (fig. 7 en bas), et comme pour ce dernier site, nous pouvons nous interroger ici sur l'éloignement de cet éventuel grenier par rapport à l'habitation: en dehors d'une zone de surveillance, proche du seul accès au site, relève-il d'un phénomène ostentatoire - la richesse est dans le grenier - ou d'une activité particulière?

\section{Autres structures}

Enfin, la localisation des bâtiments A et B dans la moitié sud de l'enclos suggère la présence d'une partition, séparant l'aire interne en deux, vraisemblablement selon un axe est-ouest, mais pas assez ancrée dans le sol pour nous être parvenue. De telles partitions se rencontrent dans les Pays-de-la-Loire, souvent sur des sites occupés plus densément, par exemple à La Chaize-le-Vicomte en Vendée (Maguer et al., 2005).

À vingt-cinq mètres au nord-est du bâtiment $\mathrm{B}$, a été mise en évidence une structure isolée de 3,50 $\mathrm{m}$ de diamètre, St. 85 (fig. 4), à proximité de la seule entrée de l'enclos. La coupe (non figurée), effectuée sur une hauteur de près de $1,50 \mathrm{~m}$, livre une stratigraphie caractéristique d'une excavation profonde : les différentes couches plongent littéralement vers son centre. Pour des raisons de sécurité, l'exploration n'a pu être prolongée qu'à l'aide d'une pelle mécanique, mais sans atteindre le fond; toutefois le bras de l'engin a pu descendre à 6,90 $\mathrm{m}$ de profondeur par rapport à la surface (cote $96,6 \mathrm{~m}$ ), de ce qui semble être un puits à eau.

\section{Hypothèses d'organisation}

L'enclos trapézoïdal du Vau Blanchard montre une occupation particulièrement faible, relevant probablement d'un unique noyau familial, modeste; ne s'y trouvent que deux bâtiments sur poteaux, à l'image de l'enclos d'Allonne dans l'Oise (Beaujard et al., 2006). On peut rappeler que, dans ce département, il a été établi une hiérarchisation entre les différents types de fermes gauloises (Malrain et Pinard,
2006, p. 244-252). Selon celle-ci, Lavernat se rapprocherait des occupations de " rang 3 ", l'expression la plus simple de l'habitat enclos. En revanche, il est difficile d'en connaître le type des occupants : des paysans libres ou une population servile relevant d'un grand propriétaire?

Le nord de l'enclos donne une impression de vide qui résulterait d'une partition et d'une spécialisation de cet espace (fig. 4). Il faut évidemment rester conscient que nombre d'occupations ou d'activités n'ont laissé aucune trace : l'érosion a entraîné la disparition des foyers de surface, des zones de travail, des jardins ou potagers, ou encore des clôtures de parcages d'animaux, pas toujours bien ancrés dans le sol. Les résultats de la fouille des structures qui ont échappé à ce sort permettent tout au plus d'esquisser quelques grandes lignes d'organisation. Les dimensions du bâtiment A dessinent un espace suffisant pour une habitation et quelques activités agricoles ( $30 \mathrm{~m}^{2}$ environ). La répartition du mobilier dans le fossé d'enclos vient conforter l'identification de cette fonction; en effet, c'est dans la partie sud-ouest de St. 1, à proximité du bâtiment $\mathrm{A}$, que fut rencontrée la plus forte densité d'un mobilier (fig. 8) dont le caractère domestique ne fait aucun doute ( $c f$. infra) : le fossé tout proche offrait une zone de rejet idéale.

Certes à un degré moindre, un phénomène identique s'observe à l'est du deuxième bâtiment mais ici, comme souvent, la présence de l'unique accès a pu également jouer un rôle de zone de rejet secondaire. La fonction retenue pour ce bâtiment $\mathrm{B}$, un grenier surélevé, expliquerait la faible quantité de tessons relevée dans cette partie orientale de l'enclos.

L'entrée de l'enclos ne paraît pas avoir bénéficié d'aménagements particuliers. Le fossé, peu marqué, s'interrompt en créant un simple passage, large de $4 \mathrm{~m}$. S'il existait une palissade, elle n'a pu être installée que sur un talus formant une petite escarpe. L'ensemble de ces observations conduit à voir une fonction défensive réduite au minimum. On n'observe toutefois pas l'aspect ostentatoire matérialisé par des fossés plus importants du côté de l'ouverture, comme parfois noté sur des enclos gaulois même peu importants (Guillier et al., 2005).

L'enclos subit un abandon, marqué par le comblement de son fossé qui disparaitt presque de la surface du sol. Certaines composantes gallo-romaines du mobilier (cf. infra), indiqueraient une mise hors fonction du site, dont les fossés devaient pourtant être encore visibles dans la première moitié $\mathrm{du} \mathrm{I}^{\mathrm{er}}$ siècle de notre ère. Cette période vit l'émergence des capitales de Cité suite à la réforme administrative engagée par Auguste. Cette nouvelle organisation eut forcément des conséquences sur les paysages, sur le peuplement et sur la nature même de l'habitat des campagnes. L'enclos du Vau Blanchard peut en constituer une modeste trace dans la nouvelle Cité des Cénomans. 


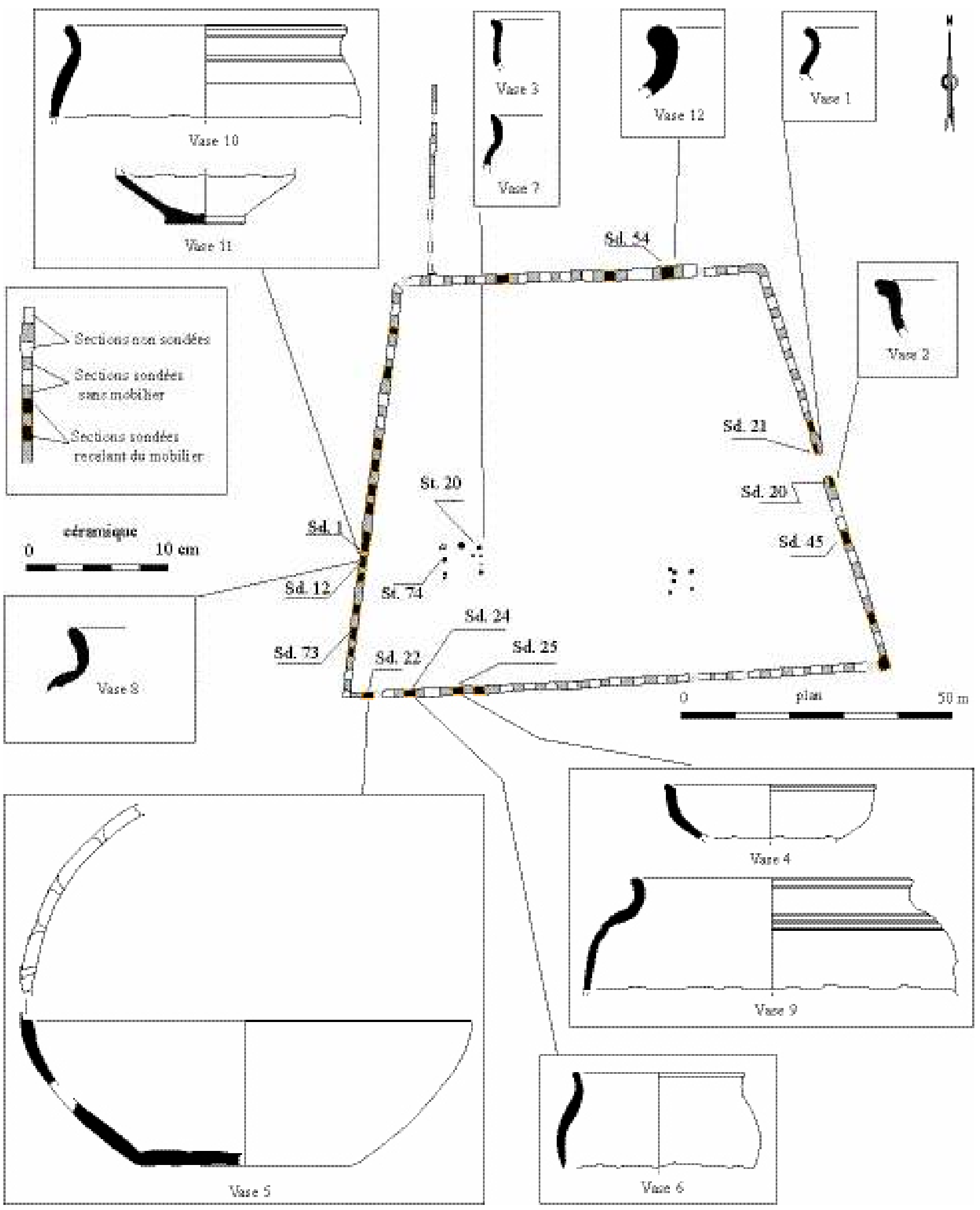

Figure 8 : Le Vau Blanchard : présentation et répartition de la céramique gauloise et antique (vase 12). Figure 8: Presentation and distribution of Gaulish ceramics, plus single Antique shard (vase 12). 


\section{LE MOBILIER ARCHÉOLOGIQUE}

\section{Présentation générale}

Le mobilier archéologique mis au jour à Lavernat est essentiellement céramique, mais il comprend aussi $11,4 \mathrm{~kg}$ d'éléments en terre cuite ${ }^{5}$; des fragments de meules, de rares objets en fer et une scorie complètent ce modeste corpus. L'occupation du site relève essentiellement de La Tène finale (388 tessons, soit $90 \%$ en nombre et $78 \%$ en poids). Son abandon au i ${ }^{\text {er }}$ siècle de notre ère est attesté par 45 tessons ( $10 \%$ en nombre et $22 \%$ en poids).

\section{Répartition et nature}

Le mobilier céramique (toutes périodes confondues) se répartit à $89 \%$ (en nombre de tessons) et $85 \%$ (en poids) dans le fossé d'enclos (St. 1), essentiellement à proximité de la zone d'habitat au sud-ouest du site (fig. 8). Pour les terres cuites, la répartition est comparable ( $86 \%$ en poids). Le reste du mobilier se localise dans le secteur des bâtiments situés à l'intérieur de l'enclos et dans le puits (St. 85). Ces chiffres désignent le fossé St. 1 comme le réceptacle principal des déchets. De tels comportements vis-à-vis des rejets se retrouvent dans l'enclos laténien d'Avrilly dans l'Eure (Guillier et al., 2005) et sur bien d'autres sites gaulois. La nature du mobilier recensé alliée à la quantité relevée de chacun de ses éléments montre leur caractère domestique : céramiques, fragments de plaques de foyer, pilettes et meules.

\section{Linstrumentum}

- Les deux meules. La meule A est constituée de la partie inférieure "dormante" d'un moulin rotatif ou meta (fig. 9 en haut). Mise au jour dans le trou de poteau $74 \mathrm{du}$ bâtiment $\mathrm{A}$, elle est de forme cylindrique, aplatie et munie d'une perforation axiale marquée d'un bourrelet en partie haute. Sa surface active, piquetée afin d'améliorer sa capacité d'abrasion, possède un poli d'usage. Son diamètre est de $360 \mathrm{~mm}$. La seconde meule, B (fig. 9 en haut), représente la partie supérieure tournante ou catillus d'un moulin rotatif. Fragmentée, elle provient à la fois du fossé d'enclos (St. 1, Sd. 73) et du trou de poteau 74 (bâtiment A). De forme cylindrique et de $380 \mathrm{~mm}$ de diamètre, elle est perforée d'un trou axial de 75 à $80 \mathrm{~mm}$ de diamètre. Sa partie supérieure offre un bouchardage grossier tandis que la surface active montre un poli d'usage. Les dimensions et la forme de ces deux éléments ne sont pas contradictoires avec une datation de La Tène finale. Malgré un matériau identique (un grès à grain fin, compact, dur, de teinte jaune-orangée), elles ne

5. Pour les éléments en terre cuite, nous raisonnons en poids et non en nombre de fragments. sont pas appariées, la meta présentant une surface active à la pente moins accentuée que le catillus. La nature des produits moulus peut être très variée, mais en contexte domestique elle se cantonnerait à des farines alimentaires (céréales, légumineuses).

- Les "terres cuites». On y distingue des fragments de plaques de foyer amovibles $(8,7 \mathrm{~kg})$, des éléments de pilettes $(1,3 \mathrm{~kg})$, des fragments d'argile rubéfiée $(1,4 \mathrm{~kg})$ et des restes de torchis.

Les plaques de foyer, très fragmentées et essentiellement issues du fossé St. 1, sont confectionnées à l'aide de plaques ou de boulettes d'argiles juxtaposées et tassées dans une forme rectangulaire. Elles contiennent un dégraissant sableux fin abondant et quelques éléments plus grossiers. Leur teinte varie du rouge orangé au brun. Elles offrent des surfaces lissées sur la partie supérieure et plus brutes de l'autre côté; de petits bourrelets en marquent la circonférence. Leur épaisseur varie de 29 à $55 \mathrm{~mm}$, mais est surtout comprise entre 31 et $45 \mathrm{~mm}$ (fig. 9, tableau). De telles pièces rectangulaires se trouvent dans une vaste zone intégrant la Loire-Atlantique, l'est du Morbihan et le sud de l'Ille-etVilaine (Le Goff, 2003). Plus près de Lavernat, signalons des découvertes dans le Maine-et-Loire (Nillesse, 2003), en Mayenne à Entrammes et dans le nord sarthois (Maguer et al., 2003), sur les sites de Vivoin. Enfin, sur le site tout proche de Beauregard à Dissé-sous-Courcillon (fig. 2, n ${ }^{\circ}$ ), il a en a été recueilli $360 \mathrm{~kg}$ (Nillesse, 2004).

Trois fragments de pilettes, constitués d'un matériau identique aux plaques de foyer ont été exhumés de la partie ouest et sud-ouest de St. 1 ; ces objets pouvaient supporter des plaques de foyer (Nillesse, 2004, p. 30-31). À Lavernat, les fragments qui nous sont parvenus (fig. 9), ont des sections variant de 5,4 x 5,5 à 6,3 x $6,6 \mathrm{~cm}$. Des vestiges comparables proviennent du site tout proche de Beauregard à Dissé-sousCourcillon (ibid., fig. 61).

- Le métal est peu représenté, comme souvent sur ce type de site (Bouvet, 2001, p. 61). Nous relevons un clou (St. 21), un petit fragment en fer très oxydé de nature indéterminée (St. 85) et un éventuel fragment de lingot, mesurant $32 \times 45 \mathrm{~mm}$, épais de $12 / 13 \mathrm{~mm}$ et pesant $100 \mathrm{~g}$ (St. 1). Mentionnons aussi une scorie de fond de forge (St. 1), en calotte plus ou moins hémisphérique, bien caractéristique (Mangin, 2004, p. 101-102). Il s'agit d'une pièce mesurant 76 × $85 \mathrm{~mm}$, d'une épaisseur de $35 \mathrm{~mm}$ et pesant $215 \mathrm{~g}$; elle est recouverte en partie supérieure d'une surépaisseur vitreuse de teinte claire.

Ces rares éléments, gaulois ou éventuellement galloromains, trahissent une économie modeste, sans aucune mesure avec celle d'autres sites régionaux où l'instrumentum occupe une place plus importante. À titre d'exemple, mentionnons le site gaulois voisin de Beauregard d'où pro- 


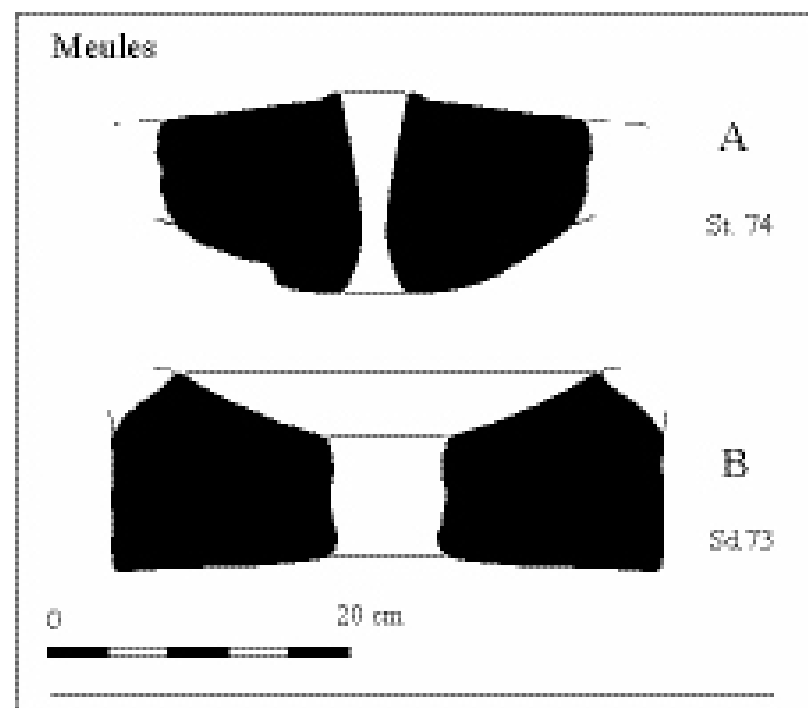

\section{Pilettes}

St 1. S4. 6?
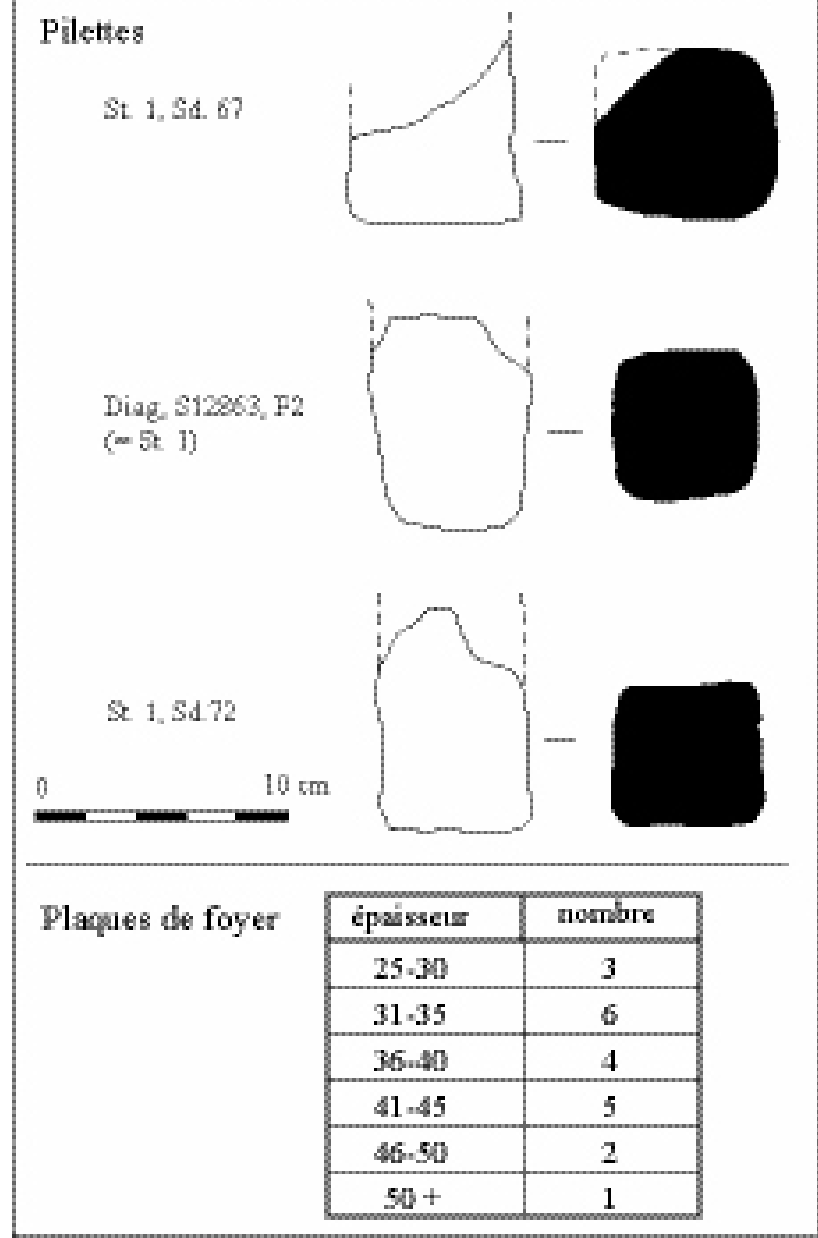

Figure 9: Le Vau Blanchard : meules, fragments de pilettes et tableau des épaisseurs (par tranches de $5 \mathrm{~mm}$ ) pour les fragments de plaques de foyer.

Figure 9: Querns, fragments of supports and table summarizing thickness of hearth slabs. viennent 208 fragments de scories pour 18,4 $\mathrm{kg}(0,2 \mathrm{~kg}$ au Vau Blanchard) et plus de 20 objets, dont des pinces, des socs d'araire, une épée, des lève-loquets... (Nillesse, 2004, fig. 62 et 63).

\section{La céramique laténienne}

Le caractère domestique de l'occupation est confirmé par le corpus des céramiques, malgré le faible nombre de vases identifiables : 17 individus au total (NMI) pour 388 tessons. Sur le plan technique, les céramiques sont modelées (7 ex.) ou tournées (5), parfois tournassées (1); des surfaces altérées n'ont pu permettre de détermination dans quatre cas. Plusieurs types de pâtes se distinguent en fonction de la technique de montage des vases. Les céramiques modelées ont des pâtes plutôt siliceuses, de teinte brune, brun-gris ou brun-noir, soigneusement lissées et de même teinte en surface et en section. Les vases tournés offrent également des pâtes siliceuses, mais à cœur gris parfois brun-noir, alors que les surfaces externes, lissées, se colorent d'orangé-grisâtre à brun-clair. Vu le faible effectif, il n'est guère possible d'envisager une typologie aboutie; aussi nous cantonnerons à une simple présentation.

La céramique de La Tène finale (fig. 8) se caractérise par la présence d'écuelles à bord déjeté, à panse carénée (vases 1,2 et 3) ou à profil " en esse » (vase 4). Notons un bol tronconique à bord droit dont le dessus de lèvre est décoré d'impressions (vase 5). Les écuelles ont une pâte fine, leurs surfaces sont soigneusement lissées, l'une d'elles porte une incision au niveau de la carène (vase 3); elles sont employées aussi bien pour la préparation et la cuisson des aliments que pour leur présentation lors des repas. Une autre catégorie de vases est constituée de pots, destinés principalement à la cuisson. Ils sont ovoïdes (vases 6, 7 et 8) ou globulaires (vases 9 et 10), à l'épaule parfois soulignée d'incisions (vase 9). Enfin, six tessons appartenant à deux amphores, dont une vinaire italique de type Dressel 1 (sans plus de précision), complètent ce corpus caractéristique de La Tène D1 et du début de La Tène D2.

Ces formes se rapprochent de celles exhumées sur le site La Tène D1 - D2 de Beauregard à Dissé-sous-Courcillon (Nillesse, 2004). Quelques-unes (vases 4 et 9) offrent des parallèles à La Tène D2 avec celles du "Grand Aunay " à Yvré-l'Evèque (fig. 2, site n 4; Vacher et Bernard, 2003).

Ce bref examen confirme le caractère domestique, peu important et vraisemblablement assez bref de l'occupation du site au cours de la fin de La Tène finale. Régionalement en effet, les sites à enclos recèlent des lots céramiques bien plus importants :

- Carquefou (Loire-Atlantique) : 1542 tessons pour un NMI de 117 vases (Le Goff, 2003, p. 108); 
- Marcé (Maine-et-Loire) : "Deffroux » (2831 tessons, NMI de 126 (Nillesse, 2003, p. 156) et "Hélouine" (3917 tessons, NMI de 116 : ibid., p. 156);

- Retiers (Ille-et-Vilaine) : 2300 tessons, NMI de 155 (Le Goff, 2003, p. 106);

- Vivoin (Sarthe), "La Pièce de Bildoux » : 2231 tessons, NMI de 161 (Maguer et al., 2003, p. 227) et « La Gaudine " : 1476 tessons, NMI de 199 (Guillier et al., 2007, p. 52);

- Dissé-sous-Courcillon (Sarthe), «Beauregard » : 7979 tessons, NMI de 122 (Nillesse, 2004, p. 20).

Les établissements ruraux fouillés exhaustivement et présentant aussi peu de mobilier que le "Vau Blanchard » sont rares. Citons le «Clos des Forges " à Avrilly dans l'Eure, qui a livré 276 tessons pour un NMI de 26 (Guillier et al., 2005) et celui de Neuville-près-Sées dans l'Orne, où 251 tessons ont été découverts (Besnard-Vauterin, 2005).

\section{Une très faible occupation gallo-romaine}

La céramique gallo-romaine n'est matérialisée que par 45 tessons (dont 43 dans St. 1). Ils ont été découverts pour $75 \%$ d'entre eux lors du diagnostic, ce qui nous suggère leur présence dans les seules couches supérieures du fossé puisque son tracé a été presque entièrement mis au jour à cette occasion. Nous avons noté des dolia (fig. 8 , vase 12 ), dont un similaire à ceux produits dans l'atelier de Mougon (Indreet-Loire), un bord de cruche, un tesson appartenant à une amphore de type Pascual 1 (Tarraconaise), dont la production supplante les amphores italiques à partir du troisième quart du Ir siècle av. J.-C. Chronologiquement, ces artefacts sont attribuables au début du $\mathrm{I}^{\mathrm{er}}$ siècle de notre ère.

À voir cette poignée de tessons, nous exprimons notre doute sur le fait qu'ils matérialisent une réelle phase d'occupation de l'enclos, même limitée. Leur dépôt dans les couches de comblement final du fossé d'enclos St. 1, alors qu'il devait être encore quelque peu visible sur le terrain, signerait plutôt l'existence d'une occupation extérieure à l'emprise de la fouille, supposant éventuellement un déplacement de l'habitat et l'apport de terre ou de fumure sur un site désormais abandonné et en voie de nivellement.

\section{ESSAI DE MISE EN PERSPECTIVE}

Si quelques informations se dégagent de ces éléments et suscitent l'intérêt de cette opération, il faut rester conscient des limites de l'exercice par rapport à une certaine déficience des données et des connaissances acquises sur le terrain au niveau du département de la Sarthe. En effet, les établissement ruraux gaulois datables de La Tène $\mathrm{C}$ ou $\mathrm{D}$, n’y sont pas nombreux : à Vivoin, «La Fosse » (Morzadec, 1999, phases 3 et 4), "La Gaudine » et "La Pièce de Bildoux » (Maguer $e t$ al., 2003; Guillier et al., 2007) (fig. 2, sites 1, 2 et 3), « Le Grand Aunay " à Yvré-l'Evêque (Vacher et Bernard, 2003) (site 4) et "Beauregard » à Dissé-sous-Courcillon (Nillesse, 2004) (site 5). Relatons aussi la découverte d'indices matérialisés par des segments de fossés accompagnés de mobilier : "Le Tertre » à Souligné-Flacé (Bouvet, 2001; site 6), «Les Petites Landes " à Teillé (ibid.; site 7), "Les Rues Vertes " à Saint-Jean-d'Assé (ibid.; site 8) et "Le Ruisseau " à Yvrél'Evêque (Guillier, 1999; site 9). En revanche, les nombreuses anomalies repérées par photographie aérienne laissent présumer un territoire densément occupé (Bouvet, 2001, p. 59), et ce probablement sur les meilleures terres de la Cité des Aulerques Cénomans comme le suggèrent les cartes de répartition des sites du Ir et du II ${ }^{\mathrm{e}}$ Âge du Fer (ibid., fig. 4 et 5), selon ce que J.-C. Meuret nomme un clivage pédogéologique (Leroux et al., 1999, p. 97).

\section{Une forme désormais classique?}

La forme trapézoïdale est courante pour les enclos de cette période (fig. 10). Mentionnons en Haute-Normandie, dans le pays de Caux, Veauville-le-Baons «RD 37 », SaintAubin-Routot "Le Four à Chaux ", Gremonville " Le Gal », Criquettot-sur-Ouville « La Folie», Baons-le-Comte « Les Baons " ou Cottevrard "La Bucaille " (Blancquaert et al., 1996; Desfossés, 1996). Nous pensons aussi aux enclos d'Avrilly (Guillier et al., 2005), de Poses (Dechezleprêtre et al., 1997, p. 50 et fig. 3), de Parville (Lukas, 2007), de Douains (Varin, 2007) ou d'Ivry-la-Bataille et de Piseux (Le Borgne et al., 2002) dans l'Eure, sur le territoire des Aulerques Eburovices. Mentionnons encore ceux de Baud et de Crédin dans le Morbihan, révélés par photographie aérienne (Leroux et al., 1999, p. 117 et 137), de Pouille en Vendée (Nillesse, 1994), ou d'Herblay dans le Val-d'Oise (Valais, 1994), qui présentent une forme géométrique similaire. En région parisienne, citons encore les Antes à Rungis dans le Val-de-Marne (Bostyn, 2002, p. 111-174) et le Plessis-Gassot dans le Val-d'Oise (Cammas et al., 2005). Plus au nord, dans l'Oise, on trouve le site des "Gâts » à Verberie, dont l'enclos offre des proportions similaires à celui du Vau Blanchard (Malrain et Pinard, 2006, p. 90-91) et celui d'Allonne près de Beauvais, où un enclos trapézoïdal est complété d'une palissade (Beaujard et al., 2006).

Avrilly, dans l'Eure, offre une nette ressemblance avec le Vau Blanchard de par sa forme, son organisation interne, la faible quantité de mobilier recueilli et sa chronologie d'occupation. Une ferme un peu plus ancienne (début de La Tène D1) y présente une organisation aussi peu dense avec un silo, un atelier enterré et surtout un bâtiment 
principal et un grenier, très semblables à ceux de Lavernat (Beaujard et al., 2006). Dans la Sarthe, sur le territoire des Aulerques Cénomans, les prospections aériennes ont livré des formes assez similaires, à Amné, Champfleur, Longnes ou René (Bouvet, 2001). Chez leurs voisins occidentaux, les Aulerques Diablintes, nous avons relevé des enclos trapézoïdaux à Chailland, Cossé-le-Vivien, Nuillé-sur-Vicoin et Saint-Berthevin (Naveau, 1992). Si cette forme est sans doute courante, en revanche les trapèzes isocèles sont rares, hormis celui de Crédin, dont les dimensions se rapprochent fort de Lavernat (fig. 10).

Par sa morphologie, l'enclos du Vau Blanchard s'insère dans un schéma qui émerge des données récemment acquises dans l'ouest de la Gaule : les "enclos trapézoïdaux à entrée axiale" (Leroux et al., 1999, p. 55-56 et fig. 31). Ce type de site est identifié par des bâtiments sur poteaux, un système d'enclos rectilignes, un plan très organisé, une faible surface enclose et une morphologie générale caractéristique. Des
Figure 10 : plans comparés d'enclos trapézoïdaux gaulois de l'ouest de la Gaule.

Figure 10:

Comparative plans of trapezoidal enclosures from Western Gaul.

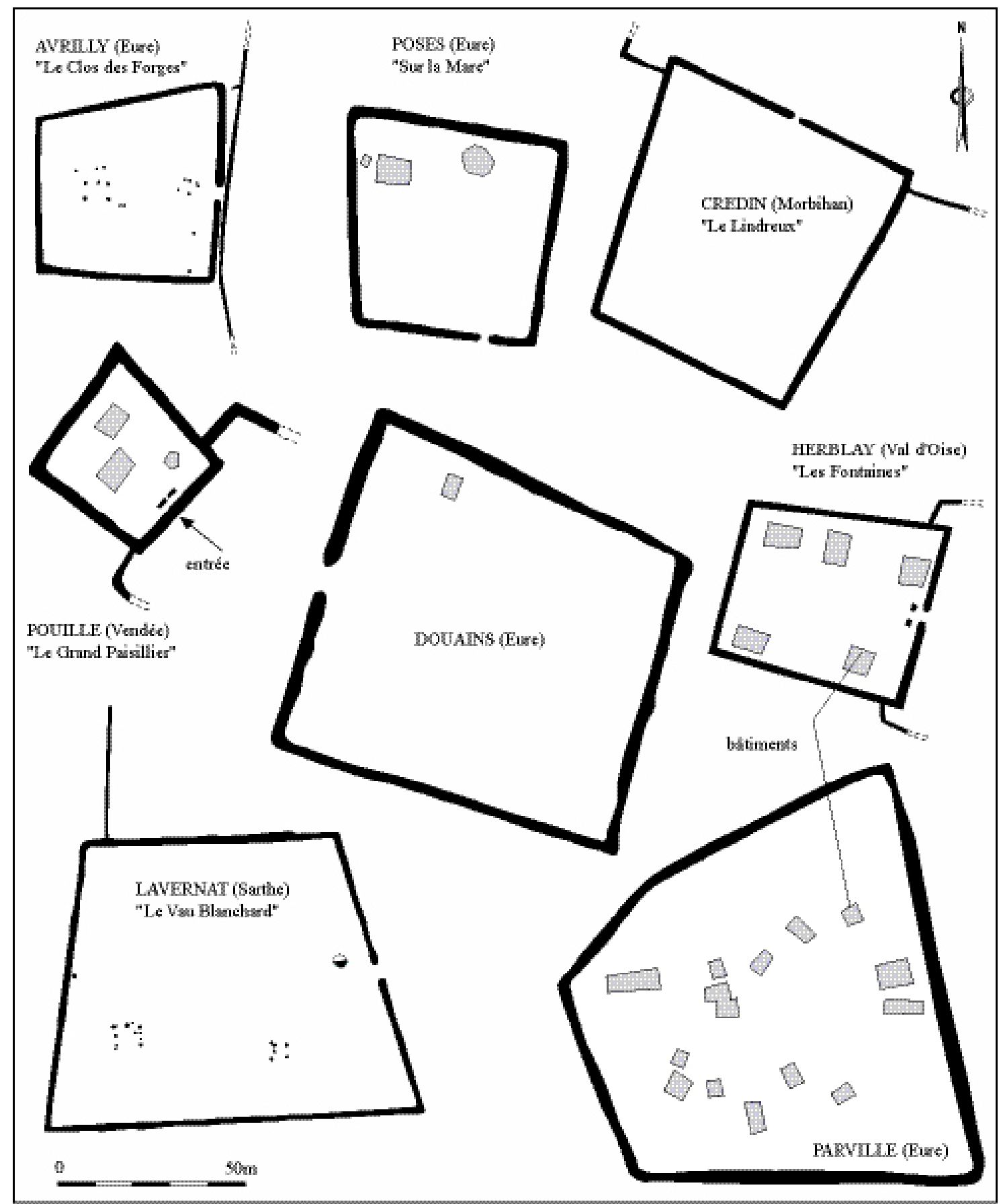


établissements aussi modestes que celui de Lavernat se rencontrent aussi, plus au nord, chez les Bellovaques (Beaujard et al., 2006), augurant d'une diffusion plus vaste de ce type d'enclos.

\section{Comparaison n'est pas déraison}

Ainsi que nous l'avons déjà souligné, de nombreux points de comparaisons existent entre les plans d'enclos trapézoïdaux (fig. 10) et les plans des structures qui les occupent (fig. 7). Si l'on associe ces convergences, il ne nous paraît pas impossible d'émettre l'hypothèse qu'il existerait - certainement au sein d'autres types -, une forme particulière (trapézoïdale), répétitive, dessinant un plan-type particulièrement adapté à son environnement naturel. L'exemple de Lavernat obéit de plus à des règles géométriques simples, faciles à mettre en œuvre sur le terrain (utilisation de rapports simples: $1 / 2,1 / 3$ et 4/3) tant pour tracer au sol le plan de l'enclos que celui des structures d'habitat, à partir d'une corde à nouds par exemple; leur évolution ultérieure dépend probablement de facteurs externes. Cette hypothèse demande à être confirmée, tout en sachant que nos informations sont grandement tributaires du hasard des recherches. L'hypothèse d'un modèle de la ferme indigène à La Tène finale n'est pas une nouveauté; elle a déjà été évoquée au sujet des enclos gaulois du pays de Caux (Desfossés, 1996, p. 205) qui, rappelons-le, sont très proches de celui du Vau Blanchard.

\section{ConClusion gÉNÉrale}

Le site du Vau Blanchard, actuellement unique dans le département de la Sarthe, devait correspondre à une petite unité agricole exploitée à La Tène finale, occupée sur un laps de temps assez court (une ou deux générations), le site acquérant sa forme définitive au moment même de sa création. Le fossé de l'enclos formait une protection surtout destinée à maintenir à distance les animaux prédateurs et à délimiter un espace utilitaire. L'occupation, comme l'a montré la fouille, est peu dense; une simple cellule familiale pouvait y être installée pour mettre en valeur un petit territoire alentours (sur un finage). Nous pouvons évoquer l'hypothèse d'un établissement de défrichement, installé sur des terres pauvres, peut-être à cause d'une pression démographique entraînant une conquête de terres nouvelles comme le souligne J.-C. Meuret (in Leroux et al., 1999, p. 98), les terres les plus riches étant déjà occupées.

L'enclos, occupé brièvement à la fin de La Tène finale, disparaît totalement dans le paysage et aucune occupation postérieure d'habitat ne s'y substitue à son emplacement direct, contrairement à nombre d'autres sites fondés à la même époque. L'existence d'un domaine dans le secteur expliquerait la désaffection de cette structure rurale inadaptée au mode d'exploitation latifondiaire, bien que la recherche récente ait pu mettre en évidence la création de petites structures gallo-romaines typologiquement proches des modèles gaulois (Guillier, 2002; Guillier et al., 2006).

Ce type de découverte contribue à nuancer l'idée d'une zone "désertique " en marge de la cité des Cénomans. À l'heure actuelle, si le site apparait comme unique localement, il y atteste néanmoins une présence humaine à l'Âge du Fer et notamment à La Tène finale. Il ne saurait être question cependant de statuer, sans autre prospection, sur l'ampleur et la nature de cette occupation à l'échelle d'un territoire. Bien que limitée, la valeur démonstrative de l'enclos du Vau Blanchard est représentative de l'état de la recherche dans ce secteur et même plus encore à l'échelle du département.

Son existence suggère une mise en valeur de terres peu occupées à partir d'un semis d'autres fermes, le tout sous la direction d'une aristocratie dont on ne sait si elle est propriétaire ou non de ces exploitations dont l'ensemble reflète cependant une société hiérarchisée cohérente. C'est à cette problématique que doivent être maintenant confrontées d'éventuelles prescriptions de fouilles sur le territoire de la commune et de celles qui l'environnent. C'est en effet la seule façon d'infirmer ou de confirmer ce qui vient d'être dit.

L'enclos étudié paraît avoir subi un nivellement au courant $\mathrm{du} \mathrm{I}^{\mathrm{er}}$ siècle de notre ère. L'hypothèse d'une nouvelle gestion de l'espace à l'échelle de la Cité par le pouvoir romain, si elle est logique et séduisante, est tributaire d'une ouverture du champ de la recherche; les prospections archéologiques sur le tracé de l'A 28, l'auront au moins initiée.

Enfin, insistons sur le fait que la fouille d'un établissement rural gaulois, même s'il ne présente que quelques structures et peu de mobilier, s'avère tout aussi nécessaire que celle d'un établissement dit aristocratique, afin de comprendre le plus finement possible les différentes composantes de la société de la fin de La Tène dans nos régions et d'appréhender le mode de vie de ses habitants.

\section{Remerciements}

Ils vont à l'équipe INRAP réunie pour la circonstance: Alain Betton (fouille), Anne Boterf(fouille), Stéphane Brousse (fouille), Fabrice Edin (fouille), Olivier Ezanno (fouille), Eric Girard (fouille), Serge Koseiv (fouille), Christian Le Goffic (fouille), Roland Le Guévelou (fouille), Frédéric Maret (fouille), Frédéric Melec (topographie), Didier Pfost (fouille et DAO) et Magali Patron (fouille). 


\section{Bibliographie}

Beaujard, S., Malrain, F. et Auxiette G., 2006 - Allonne : une ferme laténienne au cœur du territoire bellovaque, Revue archéologique de Picardie, $\mathrm{n}^{\circ}$ 3/4, 2006, p. 5-20.

Besnard-Vauterin, C.-C., 2005 - Une ferme de la fin de l'époque gauloise à Neuville-près-Sées "Les Ruisseaux » (Orne), Revue archéologique de l'Ouest, 22, p. 181-198.

Blancquaert, G., Rougier, R. et Desfosses, Y., 1996 - Les gisements protohistoriques sur le tracé de l'Autoroute A 29 (Le Havre - Saint-Saëns) - Document final de Synthèse, Rouen, SRA de Haute-Normandie, 270 p.

Bostyn, F., 2002 - Néolithique et Protohistoire du site des Antes, Rungis, Val-de-Marne. ARPEA et ARTCOM', 181 p.

Bouton, A., 1947 - Les Voies antiques, les Grands chemins médiévaux et les routes royales du Haut-Maine, Le Mans, Villaire, 226 p. et 1 pl. h.t.

Bouvet, J.-P., 2001 - La Sarthe 72 (Carte Archéologique de la Gaule), Paris, Académie des Inscriptions et Belles-Lettres, $519 \mathrm{p}$

Brodeur, J., 2006 - Lavernat (Sarthe, 72) "Le Vau Blanchard", $n^{\circ} 72160$ 0004, rapport final d'opération. Nantes, INRAP SRA des Pays de la Loire, 15 p., 18 pl., 8 fig.

Cammas, C., Marti, F. et Verdin, P., 2005 -Aménagement et fonctionnement de structures de stockage de la fin de l'âge du Fer : approche interdisciplinaire sur le site du Plessis-Gassot, Le Bois-Bouchard (Val-d'Oise), Revue archéologique du Centre de la France, suppl. 26 (XXVI ${ }^{\mathrm{e}}$ colloque de l'AFEAF, Paris et Saint-Denis), p. 33-54.

DAssier, J., 2000 - La grande Lieue gauloise, Gallia, 56 (1999), p. $285-311$.

Dechezleprêtre, T., Billard, C., Blanquaert, G., Fournier, P. et Langlois, J.-Y., 1997 - Les constructions à plan circulaire de Haute-Normandie, Revue archéologique de l'Ouest, 14, p. 49-56.

Dechezleprêtre, T., Cousyn, P., Paez-Rezende, L. et Rougier, R., 2000 - Architecture des bâtiments de l'Âge du Fer en Haute-Normandie, in Marion, S. et BlancQuaert, G. (dir.), Les installations agricoles de l'Âge du Fer en France septentrionale, Paris, Presses de l'ENS (coll. "Études d'Histoire et d'Archéologie », 6), p. 321-338.

Desfosses, Y., 1996 - L'évolution de la ferme indigène en Pays de Caux, l'apport des fouilles de l'Autoroute A 29, premiers résultats. Revue archéologique de Picardie, nº́cial 11 ("De la ferme indigène à la villa romaine ", Actes du $2^{\mathrm{e}}$ colloque AGER, Amiens, 1993), p. 203-208.

Gallien, V., 2004 - A 28, commune de Lavernat (Sarthe), lieudit "Le Vau Blanchard ", (Rapport de diagnostic), Nantes, INRAP - SRA des Pays de la Loire, 13 p.

GuIllier G., 1999 - Périphérique est du Mans. Rapport de fouille, Commune d'Yvré-l'Evêque (Sarthe), Site du Ruisseau, 72368
008 AH, Nantes, AFAN - SRA des Pays de la Loire, 18 p., 9 fig.

—, 2002 - Un établissement rural augustéen et tibérien à La Gaubretière "La Dugerie " (Vendée), Revue archéologique de l'Ouest, 19, p. 117-128.

Guillier, G., Biard, M., Cherel, A.-F., 2005 - Un atelier augustéen de taille de meules en poudingue au "Clos des Forges " à Avrilly (Eure), Revue archéologique de l'Ouest, 22, p. 199-220 et Annexe sous CD-rom : 13 p., 14 fig., 6 tab. et 6 phot.

Guillier, G., Adrian, Y.-M. et Doyen, D., 2006 - Entre Calètes et Bellovaques, les établissements ruraux gallo-romains de Mauquenchy "Le Fond de Randillon » (Seine-Maritime) : un modèle de la ferme antique?, Revue archéologique de Picardie, 1-2, p. 7-48.

Guillier, G., Auxiette, G. et Cherel, A.-F., 2007 - L'établissement rural de La Tène moyenne de la Gaudine à Vivoin (Sarthe) et ses activités de transformation, Revue archéologique de l'Ouest, 23, 2006, p. 45-70.

Le Borgne, V., Étienne-Eudier, A., Le Borgne, J.-N., Eudier, P. et Dumondelle, G., 2002 - Archéologie aérienne dans l'Eure. Paris, éd. Page de Garde, 101 p.

LE Goff, E., 2003 - «Armorique » et " Bretagne » : réflexion sur l'assimilation de ces concepts pour la fin de l'Âge du Fer. Revue archéologique de l'Ouest, suppl. $\mathrm{n}^{\circ}$ 10, p. 103-117.

Leroux, G., Gautier, M., Meuret, J.-C. et NaAs, P., 1999 Enclos gaulois et gallo-romains en Armorique, Rennes, éd. RAO (coll. «Documents archéologiques de l'Ouest»), 335 p.

LuKas, D., 2007 - Parville : de la ferme indigène à la villa galloromaine (plaquette d'exposition), Rouen, Musée départemental des Antiquités, 4 p.

Maguer, P., Cherel, A.-F. et Auxiette, G., 2003 - Nouvelles données sur les habitats de l'Âge du Fer dans le nord de la Sarthe (Commune de Vivoin, autoroute A 28), Revue archéologique de l'Ouest, suppl. $\mathrm{n}^{\circ} 10$, p. 213-234.

Maguer, P., Artuis, R. et Hiernard, J., 2005 - L'établissement rural de la Chapellière à la Chaize-le-Vicomte (Vendée) : une ferme gauloise de la fin du deuxième âge du Fer (II ${ }^{\mathrm{e}}$ siècle- ${ }^{\mathrm{er}}$ siècle a. C.), Aquitania, 21, p. 21-72.

Malrain, F. et Pinard, E., 2006 - Les sites laténiens de la moyenne vallée de l'Oise du $\mathrm{v}^{\mathrm{e}}$ au $\mathrm{I}^{\mathrm{er}} \mathrm{s}$. avant notre ère. Revue archéologique de Picardie, numéro spécial 23, 268 p.

Mangin, M., 2004 - Le fer, Paris, Errance (coll. «Archéologiques »), $239 \mathrm{p}$.

Manivit, J., Macaire, J.-J. et Maget, P., 1998 - Carte géologique de la France à 1/50000, $n^{\circ}$ 426, Château-du-Loir. Paris, BRGM, feuille et notice explicative, $63 \mathrm{p}$.

Morzadec, H., 1999 - Rapport de fouille, Commune de Vivoin, (72) "La Fosse ", 72380014 AH. Autoroute A 28, série fouilles, 22, Nantes, AFAN - SRA des Pays de La Loire, 103 p., 59 fig, L pl., annexes. 
Naveau, J., 1992 - La Mayenne 53 (Carte Archéologique de la Gaule), Paris, Académie des Inscriptions et Belles-Lettres, $176 \mathrm{p}$.

NiLlesse, O., 1994 - Les établissements ruraux gaulois dans le sud de la Vendée, in Buchsenschutz, O. et Méniel, P. (dir.), Les installations agricoles de l'Âge du Fer en Île-de-France, Paris, Presses de l'ENS (coll. Études d'Histoire et d'Archéologie, IV), p. 277-293.

—, 2003 - Les établissements ruraux gaulois de Marcé (Maine-etLoire) et la hiérarchisation des aedificia, Revue archéologique de l'Ouest, supplément $\mathrm{n}^{\circ} 10$, p. 149-172.

—, 2004 - Sarthe, Dissé-sous-Courcillon, site "Beauregard " 72115 010 1H, Etablissement rural gaulois de La Tène finale (DFS), Nantes, INRAP, SRA des Pays de La Loire, 51 p., 79 fig.

Pion, P., 1996 - Les établissements ruraux dans la vallée de l'Aisne de la fin du second âge du Fer au début du Haut-Empire romain ( $\mathrm{II}^{\mathrm{e}}$ siècle av.- $\mathrm{I}^{\mathrm{er}}$ siècle apr. J.-C.) : bilan provisoire des données et esquisse de synthèse, Revue archéologique de Picardie, $\mathrm{n}^{\circ}$ spécial 11 (" De la ferme indigène à la villa romaine », Actes du 2 colloque AGER, Amiens, 1993), p. 55-107.

VACHER, S. et Bernard, V., 2003 - Un site en zone inondable : Le Grand Aunay à Yvré-l'Évêque, Revue archéologique de l'Ouest, supplément $\mathrm{n}^{\circ} 10$, p. 189-212.

Valais, A., 1994 - La ferme des Fontaines à Herblay (Val-d'Oise), in Buchsenschutz, O. et MÉniel, P. (dir.), Les installations agricoles de l'Âge du Fer en Île-de-France, Paris, Presses de l'ENS (coll. "Études d'Histoire et d'Archéologie ", IV), p. 113-124.

Varin, W., 2007 - Douains, Normandie Parc, Zone B. Bilan scientifique 2004, Rouen, DRAC Haute-Normandie, Service régional de l'Archéologie, p. 29-30. 